\title{
Projection imaging of myocardial perfusion: minimizing the subendocardial dark-rim artifact
}

\author{
Behzad Sharif1, ${ }^{1 *}$, Rohan Dharmakumar ${ }^{1,2}$, Troy Labounty ${ }^{1}$, Chrisandra Shufelt ${ }^{1}$, Louise Thomson ${ }^{1}$, \\ Noel Bairey Merz', Daniel S Berman ${ }^{1}$, Debiao Li $\mathrm{Li}^{1,2}$ \\ From 15th Annual SCMR Scientific Sessions \\ Orlando, FL, USA. 2-5 February 2012
}

\section{Summary}

We demonstrate that projection imaging of first-pass myocardial perfusion is robust to a major cause of the so-called subendocardial dark-rim artifact (DRA); thereby proposing radial $\mathrm{k}$-space sampling as the preferred acquisition scheme for DRA-free perfusion imaging.

\section{Background}

Current methods for clinical myocardial perfusion (MP) imaging suffer from image artifacts, specifically the socalled subendocardial dark-rim artifact (DRA) [1-3]. DRAs are especially limiting since they can reduce the sensitivity/specificity of detecting subendocardial MP deficits; hence, eliminating such artifacts remains an active area of research [1]. In this work, we demonstrate that projection imaging of MP is free of Gibbs ringing [2], a major cause of DRAs for conventional schemes.

\section{Methods}

In Cartesian MP MR, Gibbs ringing along the phaseencode (PE) direction leads to DRAs due to an inherent property of the Fourier transform (FT) at sharp signal intensity transitions [2]. However, in projection imaging, the underlying data transform is the Radon transform [4], which does not exhibit the Gibbs ringing in the same form as FT. This fact is demonstrated in Fig. 1 using a numerical simulation. To investigate the validity of this observation in-vivo, healthy canines $(\mathrm{N}=2)$ and healthy human volunteers ( $N=4$; IRB approved) were imaged on a clinical 3T scanner (Siemens Verio). Two first-pass MP scans (SR-prepared FLASH) were performed at rest ( $>10$ minutes gap) using a single-shot

${ }^{1}$ Biomedical Sciences, Biomedical Imaging Research Institute, Cedars-Sinai Medical Center, Los Angeles, CA, USA

Full list of author information is available at the end of the article radial (customized) pulse sequence followed by a singleshot Cartesian (product) sequence (common parameters: FOV read $=270-300 \mathrm{~mm} ; \mathrm{BW} \approx 800 \mathrm{~Hz} /$ pixel; flip angle $=12$; $\mathrm{TR}=2.4 \mathrm{~ms} ; \mathrm{TI}=100 \mathrm{~ms}$ ). Both scans were accelerated using rate 2 self-calibrating parallel imaging with 36-40 readouts. The customized radial sequence incorporated gradient delay correction and a 4-fold interleaving scheme combined with KWIC processing [5]. Although the readout resolution for the Cartesian scan was higher than the base resolution for the radial scheme $(\approx 1.8 \mathrm{~mm}$ vs. $\approx 2.2 \mathrm{~mm})$, the overall resolution of Cartesian (PE resolution $\approx 2.6 \mathrm{~mm}$ ) and radial scans were matched to within $10 \%$.

\section{Results}

Fig. 2 shows the reconstruction results for representative $\operatorname{dog}(\mathrm{a}, \mathrm{b})$ and human $(\mathrm{c}, \mathrm{d})$ scans. Each pair of Cartesian/ radial images correspond to the same phase of the contrast uptake (late-LV/early-myocardial enhancement). As is seen in the figure, Cartesian images $(\mathrm{a}, \mathrm{c})$ show DRAs (arrows pointing to hypo-intensities) in the subendocardial regions whereas the projection reconstructions $(\mathrm{b}, \mathrm{d})$ are free of dark rims (with slightly reduced CNR).

\section{Conclusions}

Recently, a major approach for eliminating the DRAs has been to improve the spatial resolution and thereby reducing Gibbs ringing $[1,6]$. In this work, we demonstrated that projection imaging is inherently robust to Gibbs ringing. We conclude that an alternative strategy (besides increasing the resolution) is to employ projection imaging (radial trajectories). The presented results were limited to rest scans, although we expect the same properties to hold for stress imaging. However, to match the high resolutions achieved in advanced Cartesian schemes (e.g., [6]), rate 2 parallel imaging 

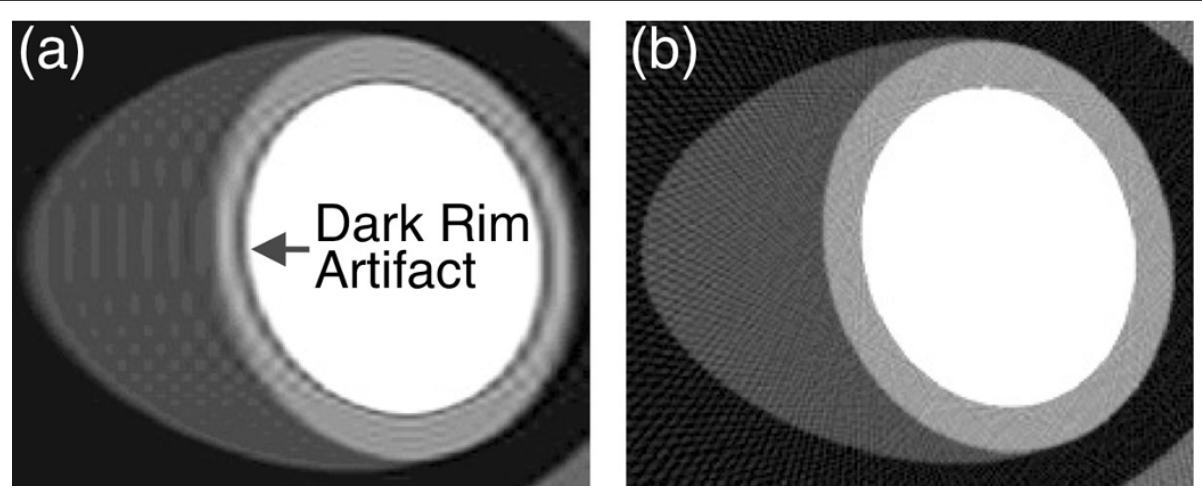

Figure 1 Numerical simulation (with realistic intensity values) demonstrating robustness of projection imaging to Gibbs ringing. (a) Cartesian imaging with 108 PEs (DRA is seen as highlighted); (b) projection imaging with 108 projections and filtered backprojection reconstruction (mild streaking artifacts are present).

(a) Healthy Dog; Cartesian

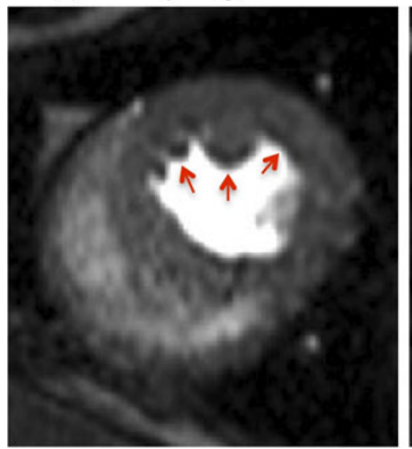

(b) Healthy Dog; Radial

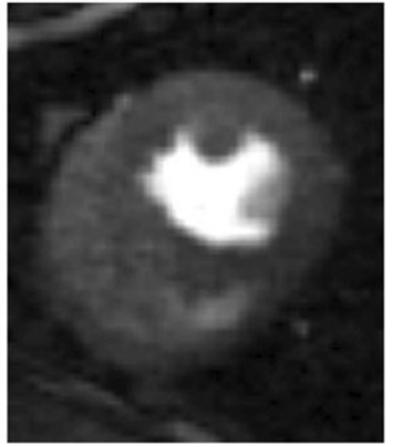

(c) Healthy Human; Cartesian

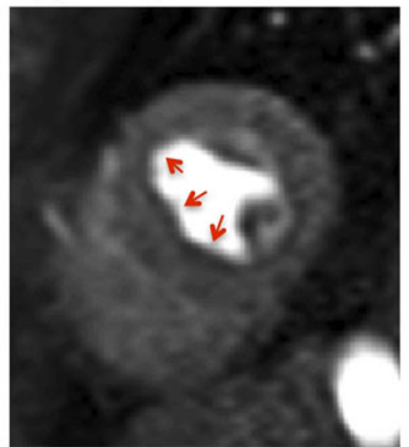

(d) Healthy Human; Radial

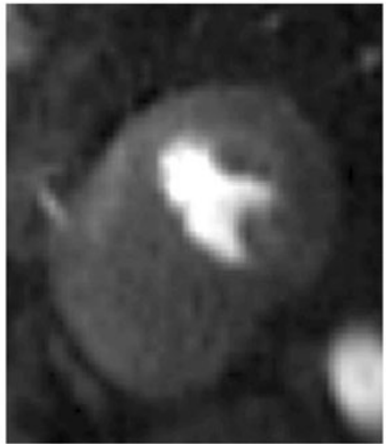

Figure 2 Representative in-vivo results for rest first-pass myocardial perfusion scans: $(a, b)$ healthy canine model; $(c, d)$ healthy human volunteer. Each pair of Cartesian/radial images corresponds to the same phase of the contrast uptake (late-LV or early-myocardial enhancement phase). The arrows in Panels (a) and (c) point to dark-rim artifacts (DRAs). However, DRAs are not seen in the projection-reconstruction (radial sampling) images in Panels (b) and (d).

acceleration is not sufficient and constrained highlyaccelerated reconstruction will be needed.

\section{Funding}

Grant sponsors: American Heart Association Postdoctoral Fellowship Award 11POST7390063 (PI: B. Sharif); National Institutes of Health grants nos. NHLBI HL38698 (PI: D. Li) and NHLBI HL091989 (PI: R. Dharmakumar).

\section{Author details}

${ }^{1}$ Biomedical Sciences, Biomedical Imaging Research Institute, Cedars-Sinai Medical Center, Los Angeles, CA, USA. ²Bioengineering, UCLA, Los Angeles, CA, USA.

Published: 1 February 2012

\section{References}

1. Gerber, et al: JCMR. 2008, 10:18.

2. Di Bella, et al: MRM. 2005, 54:1295.

3. Ferreira, et al: JCMR. 2009, 11:17.
4. Kak, Slaney : IEEE press. 1988

5. Peters, et al: MRM. 2006, 55:1150.

6. Plein, et al: MRM. 2007, 58:777.

doi:10.1186/1532-429X-14-S1-P275

Cite this article as: Sharif et al:: Projection imaging of myocardial perfusion: minimizing the subendocardial dark-rim artifact. Journal of Cardiovascular Magnetic Resonance 2012 14(Suppl 1):P275.

\section{Submit your next manuscript to BioMed Central} and take full advantage of:

- Convenient online submission

- Thorough peer review

- No space constraints or color figure charges

- Immediate publication on acceptance

- Inclusion in PubMed, CAS, Scopus and Google Scholar

- Research which is freely available for redistribution 